\title{
Modification of Edmonds' Maximum Matching Algorithm*
}

\author{
C. Witzgall and C. T. Zahn, Jr. ${ }^{* \star}$
}

\begin{abstract}
(November 16, 1964)
Edmonds developed an efficient algorithm for finding in a given graph $G$ a matching of maximum cardinality. This algorithm "shrinks" parts of the graph $G$. Although helpful to the intuitive understanding of the theory, shrinking is complicated to implement on an elêctronic computer. The modification presented in this paper avoids shrinking. It employs instead a treelike arrangement of alternating paths. The possibility of such an arrangement is also of theoretical interest, and its proof forms the main part of the paper.
\end{abstract}

\section{Introduction}

A matching $M$ in a graph $G$ is a set of edges of $G$ such that no two meet the same vertex. Edmonds $[1]^{1}$ developed an efficient algorithm for finding in a given graph $G$ a matching of maximum cardinality. The maximum matching problem belongs to a class of problems which for the most part have defied efficient treatment. These are integer linear programming problems-in particular those associated with networks. A celebrated and still largely intractable representative of this class is the traveling salesman problem.

The techniques on which Edmonds bases his algorithm have some interesting theoretical consequences. For example, Edmonds assigns the labels "outer" and "inner" to the vertices of the graph $G$. This assignment is based on the underlying matching; however, it turns out to be the same for all maximum matchings. Therefore the "inner" and "outer" property is an invariant of the graph itself. The outer vertices (with respect to any maximum matching) are precisely those vertices which are "exposed"-that is, they meet no matched edge-in some maximum matching of the graph. The set of outer vertices is of considerable structural interest (compare Edmonds [1]).

The notion of outer vertices is closely related to accessibility by simple alternating paths. To clarify this statement, we need some definitions.

First we note that it involves no loss of generality when searching for a maximum matching to restrict our considerations to graphs whose edges can be conceived of as unordered pairs of vertices. Indeed, if the graph $G$ possesses pairs of vertices that are connected by a bundle of more than one edge, we construct a subgraph $\hat{G}$ by deleting from every such bundle all but one of its edges. Every maximum matching of $\hat{G}$ is also a maximum matching of $G$.

* Supported by the Army Research Office (Durham).

** Present address: General Electric Computer Department, 679 Leesburg Pike, Falls Church, $\mathrm{V}$ a.

${ }^{1}$ Figures in brackets indicate the literature references at the end of this paper.
Due to the absence of multiple edges, we may characterize paths by sequences of vertices $\left(v_{1}, \ldots\right.$, $v_{n}$ ) every two consecutive ones of which are adjacent. A path is simple if its vertices, and therefore its edges, are distinct. A closed path is simple if its vertices and its edges are distinct with the only exception $v_{1}=v_{n}$. Every path forms a subgraph consisting of the vertices $v_{i}$ of the path and the edges joining its successive vertices. A simple closed path, for instance, forms a circuit, that is, a connected graph each of whose vertices is incident to exactly two vertices.

A graph $G$, along with a matching $M$, will be called a matched graph $(G, M)$. The set of edges of $G$ not in $M$ will be denoted by $\bar{M}$. An alternating path in $(G, M)$ is a path whose successive edges are alternately in $M$ and $\bar{M}$. In particular, the path of one node is an alternating path. An exposed vertex in $(G, M)$ is one which is incident to no edges in $M$. An augmenting path is a simple alternating path connecting two exposed vertices. The significance of augmenting paths for matchings is demonstrated by the theorem of Berge [4]: A matching $\mathrm{M}$ is of maximum cardinality if and only if there exists no augmenting path in $(G, M)$. The easier part of this theorem is that, in the presence of an augmenting path, a matching cannot be maximum. Indeed, by changing all nonmatched edges of an augmenting path into matched edges and vice versa, the cardinality of the matching is increased.

According to the theorem of Berge, the maximum matching problem can be solved by searching for all alternating paths from each exposed vertex. However, one can do better. To see this we reformulate Berge's theorem, defining a vertex $v$ to be an outer vertex rooted at $e$ if $e$ is an exposed vertex which is linked to $v$ by a simple alternating path of even length (=number of edges). (In particular, we regard all exposed vertices as outer.) The reason for considering outer vertices becomes evident if one examines an augmenting path connecting two exposed vertices $e_{1}$ and $e_{2}$. Let $v_{1}$ and $v_{2}$ be the neighbors of $e_{1}$ and $e_{2}$ within the augmenting path. Then $v_{2}$ is an outer 
vertex rooted at $e_{1}$, and $v_{1}$ is an outer vertex rooted at $e_{2}$. Therefore, a matching is maximum if and only if no exposed vertex e is adjacent to an outer vertex which is rooted at an exposed vertex different from e.

For the purpose of establishing maximality or nonmaximality it is therefore sufficient to search for all outer vertices. This is a genuine improvement over searching for all alternating paths, since there are in general more alternating paths emanating from exposed points than there are outer vertices. Moreover, a list of vertices is easier to handle and store than a list of paths.

The method of Edmonds, as well as its modification described in this paper, searches for outer vertices. It should be realized, however, that if the matching turns out to be not maximum, that is, if there are outer vertices adjacent to exposed vertices other than their roots, then we still face the problem of actually finding an augmenting path. This means essentially that we should be able to retrieve for each outer vertex a simple alternating path that leads back to a root. The difference between Edmonds' method and our modification lies in the kind of additional information furnished to permit this "back-tracing". ${ }^{2}$ Edmonds shrinks the graph in such a manner that at each outer vertex in the shrunken graph there is only one possibility for stepping backwards along an alternating path: one is forced to proceed in the right direction. The modification presented in this paper is based on the notion of a "predecessor." It should be noted that in general the two methods will find different backpaths. When expanded into paths of the original graph, the back-paths defined by Edmonds' method will in general not display the treelike arrangement described in the next section.

${ }^{2}$ Rather surprisingly, in both methods the process of finding the outer vertices also requires back-tracing from outer vertices already found. The importance of back-tracing is therefore not restricted to augmentation. This "back-tracing" should not be confused, however, with so-called "back-tracking" methods that usually involve an unpleasant amount
of trial and error. Our algorithm, as well as Edmonds", is a good one.

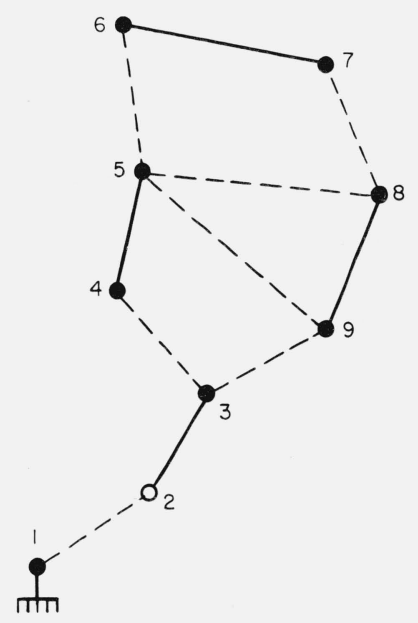

FIGURE 1. $p(3)=1, p(5)=3, p(7)=5, p(9)=7, p(8)=3, p(6)=3$, \begin{tabular}{c}
$p(6)=8, p(4)=6$. \\
\hline , Matched edge. \\
Outer points.
\end{tabular}
We assign to each nonexposed outer vertex $v$ another outer vertex $u=p(v)$ which is connected to $v$ by an alternating path of length two. Vertex $u=p(v)$ is called the predecessor of $v$. We shall prove that this assignment of predecessors can be specified in such a way that the alternating path formed by consecutive predecessors is simple and joins $v$ to an exposed vertex. (This is what we mean in saying a complete set of alternating back-paths has been arranged in a treelike manner.) We employ the fact that an alternating path is uniquely determined if only every other vertex is given.

As an example consider the graph in figure 1. The vertices are numbered $1, \ldots, 9$. Heavy lines distinguish the matched edges. Vertex $l$ is the only exposed vertex. As an augmenting path would require two exposed vertices, the matching is maximum. Solid points designate outer vertices. Vertex 2 is not outer, since every alternating path of even length joining vertex 2 to the "root", as for instance $(2,3$, $9,8,5,4,3,2,1)$, passes the edge $(2,3)$ twice, thus being not simple.

The assignment of predecessors $p(v)$, as specified in figure 1, yields a simple back-path for each outer vertex. Vertex 7, for instance, is joined to vertex $l$ by the path $(7,6,5=p(7), 4,3=p(5), 2,1=p(3))$.

\section{Graph of Bi-edges}

The situation is best understood by considering the graph of bi-edges (Edmonds [5]) which is associated with the matched graph $(G, M)$. A bi-edge is an alternating path of length two, that is, a path consisting of an unmatched edge followed by a matched one. Bi-edges are the units of which even-length alternating paths are composed. The bi-edge graph $B(G, M)$ is defined over the same vertex set as $G$, two vertices being linked if they are the end-points of a bi-edge in $(G, M)$. We regard bi-edges as directed: they lead from the end that is incident with the matched part to the end that is incident with the unmatched part of the bi-edge (fig. 2). Consequently, the graph of bi-edges is a "directed" graph. A directed graph consists of vertices, and edges which are ordered pairs of the vertices. According to this definition, a directed graph can have two edges, $\left(v_{1}, v_{2}\right)$ and $\left(v_{2}, v_{1}\right)$, incident to the same pair of vertices. Figure 3 shows the graph of bi-edges that corresponds to the matched graph in figure 1 .

A path in a directed graph can be characterized again by a sequence of vertices $\left(v_{1}, v_{2}, \ldots, v_{n}\right)$. However, we insist that each linking edge lead from a vertex to its successor in the path; more precisely, we require the edge that links two successive vertices $v_{i}$, $v_{i+1}$ to be the edge $\left(v_{i}, v_{i+1}\right)$ in this order. Each path in the bi-edge graph $B(G, M)$ corresponds to a unique alternating path in $(G, M)$. We shall say that a path in $B(G, M)$ expands into the corresponding alternating path in $(G, M)$. Alternating paths gained by expansion of bi-edge paths have even length (= number of edges, where each edge counts as often as it is traversed). 


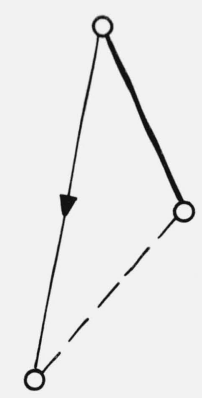

FiguRE 2

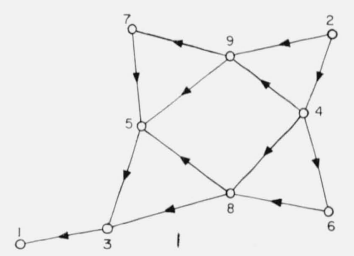

Figure 3

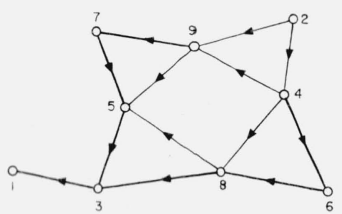

FIGURE 4
The relation between the above theorem and the algorithm for maximum matchings that will be proposed in this paper is as follows: generality of the theorem is not restricted by assuming that the root $r$ is an exposed vertex. Indeed, if $r$ meets a matched edge, then removing this edge from the matching does not change the set of simple alternating paths emanating from $r$ along nonmatched edges. The problem then becomes to arrange into a tree $T \subseteq B(G, M)$ alternating paths which connect outer vertices to an exposed vertex $r$. Our algorithm $\mathfrak{I}$ will sucessively construct this tree, thereby proving the theorem. On the other hand, the algorithm will rely at each step on the fact that the alternating paths which connect the outer vertices established at this moment to the root $r$ are properly arranged in a tree-like manner. A combination of the algorithm $\mathfrak{I}$ with augmentation using augmenting paths gives the algorithm $\mathfrak{M}$, which yields a maximum matching.

\section{Labeled Subgraphs}

Let $r$ be an exposed vertex in a matched graph $(G, M)$. We define a "labeled subgraph" with the root $r$ to be a quadruple

$$
L=(A, \Omega, I, p)
$$

where $A$ is a subgraph of $G$. The vertices of $A$ are labelled either "outer" or "inner", $\Omega$ denoting the set of vertices labeled "outer", and $I$ the set of vertices labeled "inner". $\Omega$ and $I$ are disjoint. Finally, $p$ is a single-valued function $p: \Omega-\{r\} \rightarrow \Omega$ called the predecessor function.

In addition, we shall require certain relations to hold between the elements of the quadruple $(A, \Omega$, $I, p)$. To formulate these requirements, and for later use, we introduce the following matching function $\mathrm{m}$, which maps the set of all nonexposed vertices of $G$ into itself, and which is defined by

$$
m(v):=u \quad \text { if and only if } \quad(v, u) \in M .
$$

The requirements for a quadruple $(A, \Omega, I, p)$ to be a labeled subgraph then are:

(i) If one vertex of a matched edge is in A then the entire edge is in A. 
(ii) If $\mathrm{x} \in \mathrm{I}$ then $\mathrm{m}(\mathrm{x}) \in \Omega$

(iii) The edge $(\mathrm{m}(\mathrm{v}), \mathrm{p}(\mathrm{v}))$ is in A for every $\mathrm{v} \epsilon \Omega-\{\mathrm{r}\}$ Note that (ii) and (iii) imply that $I$ and $\Omega-\{r\}$ consist of nonexposed vertices. It also follows that for $x \in I$, $(x, p m(x))$ must be in $A .^{3}$

(iv) Decomposition: If $\mathrm{x} \in \mathrm{I}$ then deleting the edge $(\mathrm{x}, \mathrm{pm}(\mathrm{x}))$ decomposes $\mathrm{A}$ into two connected components.

(v) Legality: For each vertex $\mathrm{v} \in \Omega$ the sequence of vertices $\left(\mathrm{v}, \mathrm{p}(\mathrm{v}), \mathrm{p}^{2}(\mathrm{v}), \ldots\right)$ eventually contains $\mathrm{r}$, and the back-path

$$
\mathrm{P}(\mathrm{v}):=\left(\mathrm{v}, \mathrm{m}(\mathrm{v}), \mathrm{p}(\mathrm{v}), \mathrm{mp}(\mathrm{v}), \mathrm{p}^{2}(\mathrm{v}) \ldots, \mathrm{p}^{\mathrm{n}}(\mathrm{v})=\mathrm{r}\right)
$$

has no double vertices.

The back-path $P(v)$ lies entirely in $A$ according to (i) and (ii).

The vertices of the form $p^{k}(v)$, including the vertex $v$ itself, are said to be even in $P(v)$, the rest being odd. The even vertices, in particular the root $r$, are of course in $\Omega$. The sequence of even vertices in a back-path defines a simple and legal path in the graph of bi-edges $B(G, M)$.

By virtue of the recursive structure of the backpaths, we have $P(u) \subseteq P(v)$ if $u$ is even in $P(v)$. Therefore we may define a partial ordering in $\Omega$ by letting:

$$
u \leqslant v \text { if and only if } u \text { is even in } P(v) \text {. }
$$

Any two vertices $u$ and $v$ in $\Omega$ have at least one common lower bound. By finiteness, they have greatest common lower bounds. Let $w$ and $\bar{w}$ be such greatest common lower bounds. Since $w \leqslant u$ and $\bar{w} \leqslant u$, both $w$ and $\bar{w}$ are even in $P(u)$, and therefore comparable. Hence $\bar{w}=w$ because they are both maximal. It follows that the greatest common lower bound $w=u \cap v$ is unique.

We shall now describe two operations which enlarge labeled subgraphs. If $L$ is a labeled subgraph, and there is a bi-edge $(v, x, u)$ with $u \epsilon \Omega, x \xi \Omega \cup I,(v, x) \epsilon M$, then $v \in \Omega \cup I$ by (i). We obtain a new quadruple $\tilde{L}=(\tilde{A}, \tilde{\Omega}, \tilde{I}, \tilde{p})$ by letting $\tilde{A}:=A \cup(v, x) \cup(x, u), \tilde{\Omega}$ : $=\Omega \cup\{v\}, \tilde{I}:=I \cup\{x\}$, and

$$
\tilde{p}(z):=\left\{\begin{array}{l}
p(z) \text { for } z \epsilon \Omega \\
u \text { for } z=v
\end{array}\right.
$$

\footnotetext{
${ }^{3}$ We write $p m(v)$ for $p(m(v)), m p(v)$ for $m(p(v)), p^{2}(v)$ for $p(p(v))$.
}

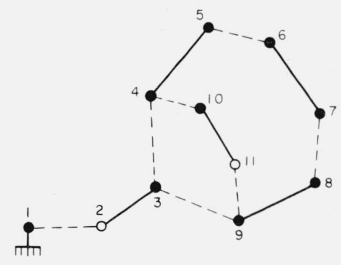

Figure 5
In other words, we add the bi-edge $(v, x, u)$ to $A$, labeling $v$ "outer" and $x$ "inner", and putting $\tilde{p}(v)$ : $=u$. The predecessor function is not changed for the old $\Omega$. Clearly, $L$ is a labeled subgraph. We write

$$
\tilde{L}:=F(L)
$$

to indicate that $\tilde{L}$ is obtained by a forward step.

If $L$ is a labeled subgraph, and there is an edge $(s, t)$ which is not in $A$ but joins two vertices of $\Omega$, then again we can enlarge the subgraph: Since $s$ and $t$ belong to $\Omega$, and $L$ is legal, we have back-paths $P(s)$ and $P(t)$. Let $b:=s \cap t$ denote the greatest common lower bound of $s$ and $t$ with respect to the ordering defined above. Then $P(s) \supseteq P(b)$ and $P(t)$ $\supseteq P(b)$. The paths $P(s)-P(b)$ and $P(t)-P(b)$ together with $b$ form a path $P(s, b, t)$ joining $s$ and $t$. Following Edmonds [1], we call this path a blossom with the base $b$ (see fig. 5). We shall see later that all vertices of a blossom are indeed legally accessible: the inner vertices, for instance in $P(s)-P(b)$, are joined to $t$ by an even alternating path, which will be shown to be simple. Labeling the inner vertices in a blossom "outer", and adjoining $(s, t)$ to $A$ is called a blossom step ${ }^{4}$

$$
\tilde{L}:=B(L)
$$

The details of a blossom step are as follows: Let $\bar{s}$ be defined as the greatest even vertex in $P(s)$ such that $P(\bar{s})-P(b)$ contains no inner vertex, and let $\bar{t}$ be similarly defined. Either $\bar{s}=s$, or the vertex $x \in P(s)$ $-P(\bar{s})$ which is next to $\bar{s}$ in $P(s)$ is in $I$. If $\bar{s} \neq s$, then let $u_{1}:=x$, and let $u_{k}$ be defined inductively as the unique vertex of $P(s)$ such that $p m\left(u_{k}\right)=m\left(u_{k-1}\right)$ until some $u_{m}=m(s)$. Put the set $\left\{u_{i} \mid \mathrm{i}=1, \ldots, m\right\}$ into $\Omega$, deleting vertices from $I$ whenever necessary, and define the new predecessor function by $\tilde{p}\left(u_{i}\right)$ $=u_{i+1}$ for $1 \leqslant i \leqslant \mathrm{~m}$ where $u_{m+1}=t$. Notice that some $u_{i}$ may be in $\Omega$ and already have a predecessor which is hereby changed. The same procedure is repeated for $t$ in place of $s$, and then we add the edge $(s, t)$ to $A$ to obtain the new graph $A$ : If the counterparts of the $u_{i}$ in the path $P(t)$ are denoted $v_{j}$ then formally we get $\tilde{A}:=A \cup(s, t), \tilde{\Omega}:=\Omega \cup\left\{u_{i}\right\} \cup\left\{v_{j}\right\}$, $\tilde{I}:=I-\left\{u_{i}\right\}-\left\{v_{j}\right\}$,

$$
\tilde{p}(z):=\left\{\begin{array}{l}
u_{i+1} \text { for } z=u_{i} \\
v_{j+1} \text { for } z=v_{j} \\
p(z) \text { otherwise. }
\end{array}\right.
$$

The above definition of $\tilde{p}(z)$ implicitly requires the sets $\left\{u_{i}\right\}$ and $\left\{v_{j}\right\}$ to be disjoint. Since the $u_{i}$ are

\footnotetext{
${ }^{4}$ In practice one will use a more elaborate blossom step. Let $x$ and $y$ be two adjacent vertices of the blossom $P(s, b, t)$. After execution of the blossom step defined by $s$ and $t$, both $x$ and $y$ will be outer whereas their connecting edge $(x, y)$ need not be in $A$. In this case, $x$ and $y$ will give rise to another blossom-step. The corresponding blossom, however, is easily verified to be contained in the circuit formed by $P(s, b, t)$ and the edge $(s, t)$. This blossom therefore contains no inner vertices, and all the blossom step does is adjoin the edge $(x, y)$ to $A$. The more elaborate blossom step consists of adding not only
} the edge $(s, t)$ but all other "diagonals" of the blossom $P(s, b, t)$ to $A$. 
odd in $P(s)$ and the $v_{j}$ are odd in $P(t)$, this will be a consequence of the following

LEMMA 1. Let $\mathrm{s}$ and $\mathrm{t}$ be vertices in $\Omega$, and put $\mathrm{b}:=\mathrm{s} \cap \mathrm{t}$. If the vertex $\mathrm{v}$ is of equal parity in both $\mathrm{P}(\mathrm{s})$ and $\mathrm{P}(\mathrm{t})$, then $\mathrm{v} \epsilon \mathrm{P}(\mathrm{b})$.

Proof: If $v$ is even in both $P(s)$ and $P(t)$, then $v \leqslant s$ and $v \leqslant t$. Hence $v \leqslant s \cap t=b$, which implies $v \epsilon P(b)$ by definition of the partial ordering.

If $v$ is odd in both $P(s)$ and $P(t)$, then $v$ cannot be the root $r$, because $r$ is even in both $P(s)$ and $P(t)$. Hence $m(v)$ is defined, and it is even in both $P(s)$ and $P(t)$. By the preceding argument we then conclude $m(v) \epsilon P(b)$ and therefore $v \epsilon P(b)$.

Before proving that the blossom step $B$ as defined above always yields a new labeled subgraph $\tilde{L}$, we illustrate the blossom step by two examples.

The first example (fig. 5) shows that the two halfs of the blossom, $P(s)-P(b)$ and $P(t)-P(b)$, need not be disjoint.

Starting with the root $r=1$, the assignment of predecessors has progressed as follows:

$$
\begin{aligned}
& \mathrm{p}(3)=1 \text { forward step } \\
& p(5)=3 \text { forward step } \\
& p(7)=5 \text { forward step } \\
& p(9)=7 \text { forward step } \\
& p(8)=3 \\
& \begin{array}{l}
p(6)=8 \\
p(4)=6
\end{array} \\
& p(10)=9 \text { forward step }
\end{aligned}
$$

Now a blossom step is due for $s=10$ and $t=4$. The back-paths are

$$
\begin{aligned}
& P(s)=(10,11,9,8,7,6,5,4,3,2,1) \\
& P(t)=(4,5,6,7,8,9,3,2,1),
\end{aligned}
$$

$b=3$ is the base. It is seen that the part $(4,5,6,7,8$, 9) of the blossom is traversed by $P(s)$ as well as $P(t)$, however in opposite directions. Changing predecessors in the area common to $P(s)$ and $P(t)$ would clearly destroy legality. This is one reason for changing predecessors only up to the last inner vertex. Later on we shall indeed see that $P(s)-P(\bar{s})$ and $P(t)$ $-P(\bar{t})$ are always disjoint.

Even if the blossom has no double points, legality may be destroyed if predecessors are changed beyond the last inner vertex. This is shown by our second example (fig. 6).

Starting with the root $r=1$, the assignment of predecessors has progressed as follows

$$
\begin{aligned}
& p(3)=1 \text { forward step } \\
& p(5)=3 \text { forward step } \\
& \left.\begin{array}{l}
p(4)=1 \\
p(2)=4
\end{array}\right\} \text { blossom step }(5,1) \text {, base } 1 \\
& p(7)=2 \text { forward step } \\
& p(8)=7 \text { forward step } \\
& p(9)=7 \text { blossom step }(8,7) \text {, base } 7 \text {. }
\end{aligned}
$$

Again a blossom step is due for $s=9$ and $t=4$. The

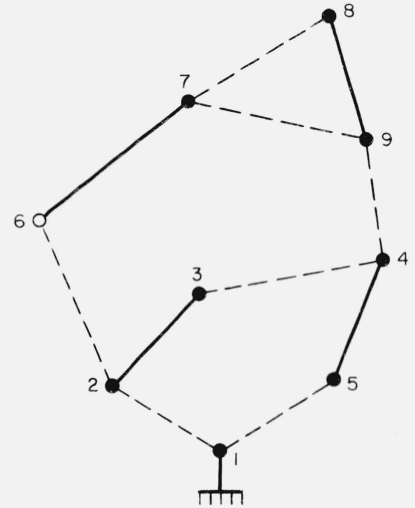

FigURE 6. - Matched edges; outer points.

back-paths are

$$
\begin{aligned}
& P(s)=(9,8,7,6,2,3,4,5,1) \\
& P(t)=(4,5,1),
\end{aligned}
$$

yielding $b=4$ as base. The blossom step as defined before puts

$$
\begin{aligned}
& \tilde{p}(8)=4 \\
& \tilde{p}(6)=8 .
\end{aligned}
$$

Note that the predecessor of 8 has been changed from 7 to 4 . If one would change the predecessor beyond the inner point 6 , that is, if one puts

$$
\tilde{p}(3)=6
$$

then the new back-path $\tilde{P}(5)$ will not be simple.

\section{Preservation of Legality}

It must he shown that $F(L)$ and $B(L)$ are again labeled subgraphs. Verifying (i) to (iii) can be left to the reader. Proving the decomposition property (iv) and, in particular, the legality (v) is the main burden of this paper.

LEMMA 2. The decomposition property (iv) is preserved by the operations $\mathrm{F}$ and $\mathrm{B}$.

Proof: In the case of a forward step $F$ there is really nothing to prove since no new circuits are introduced in $A$. In the case of a blossom step, however, new circuits are generated, and we have to show that these circuits contain no inner vertices.

Let then $s$ and $t$ be two adjacent outer vertices determining a blossom $P(s, b, t)$ with $b:=s \cap t$, and execute the corresponding blossom step. Assume that the new subgraph $\tilde{A}$ contains a circuit $C$ with a vertex $x \epsilon \tilde{I}$. Since $L$ satisfies the decomposition property (iv) and since $x \xi \Omega, C$ cannot be a circuit in $A$, and must therefore contain the new edge $(s, t)$. Replacing this edge by the blossom path $P(s, b, t)$ yields a closed path $C^{*}$ (not necessarily a circuit), which lies entirely in the old subgraph $A$, and which still contains the inner 
vertex $x$. Since $P(s, b, t) \cap \tilde{I}=\phi$ whereas $x \epsilon \tilde{I}$, the vertex $x$ is not a double point of $C^{*}$. Hence $X$ is contained in a subcircuit ${ }^{5}$ of $C^{*} \subseteq A$, contradicting (iv).

LEMMA 3. The legality property $(v)$ of a labeled subgraph $\mathrm{L}$ is preserved by the operations $\mathrm{F}$ and $\mathrm{B}$.

PRoOF: In the case of forward steps $F$, the proof presents no difficulties, and may be left to the reader. Let then $s$ and $t$ be again two adjacent vertices in $\Omega$ such that the edge $(s, t)$ is not in $A$, and execute the corresponding blossom step $L:=B(L)$. Define for $v \in \tilde{\Omega}$ the-possibly infinite-sequence

$$
\tilde{P}(v):=\left(v, m(v), \tilde{p}(v), m \tilde{p}(v), \tilde{p}^{2}(v), \ldots . .\right) .
$$

It is to be shown that $\tilde{P}(v)$ is a simple alternating path connecting $v$ to the root $r$. Trivially, this is the case if $\tilde{\boldsymbol{P}}(v)=\boldsymbol{P}(v)$. Otherwise, let $w$ be the even vertex in $\tilde{P}(v)$ that is closest to $v$, and that is odd in $P(s)$ $-P(\bar{s})$ or $P(t)-P(\bar{t})$. Since predecessors are not altered outside the set of odd vertices of $\{P(s)-P(\bar{s})\}$ and the set of odd vertices of $\{P(t)-P(\bar{t})\}$, we have

$$
\tilde{P}(v)-\tilde{P}(w)=P(v)-P(w) .
$$

Without loss of generality we may assume that $w \epsilon P(s)-P(\bar{s})$. It follows again by the definition of the blossom step $B$ that the sequence $\left(v, \tilde{p}(v), \tilde{p}^{2}(v)\right.$, ...) of even vertices of $\tilde{P}(v)$ contains $t$. Therefore $\tilde{P}(v)$ consists of three pieces, the first of which may be empty:

$$
\tilde{\boldsymbol{P}}(v)=(\tilde{\boldsymbol{P}}(v)-\tilde{P}(w), \tilde{P}(w)-\tilde{P}(t), \tilde{P}(t)) .
$$

The proof that $\tilde{P}(v)$ is simple relies on the decomposition property (iv). The vertex $x$, which precedes $\bar{s}$ in $P(s)$, is an inner point $x \in I$. According to (iv), deletion of the edge $(\bar{s}, x)$ decomposes $A$ into two components $H_{x}$ and $H_{r}$ the latter of which contains the root $r$.

We note that $x \in I$ does not occur in $P(t)$. Otherwise, it would be odd in both $P(s)$ and $P(t)$, and lemma 1 would imply $x \in P(b)$. This contradicts $x \in P(s)-P(\bar{s})$ $\subseteq P(s)-P(b)$. Since $x \notin P(t)$, the path $P(t)$ cannot enter the component $H_{x}$ because by (iv) every path from $H_{x}$ to $r$ passes through $x$. Thus

(vii)

$$
P(t) \subseteq H_{r} .
$$

Further we note that

$$
P(s)-P(\bar{s}) \subseteq H_{x} .
$$

Indeed, since $P(s)$ is simple, the edge $(\bar{s}, x)$ occurs exactly once in $P(s)$, bisecting this path. The piece $P(s)-P(\bar{s})$ lies in $H_{x}$, the piece $P(\bar{s})$ in $H_{r}$.

It follows from (vii) and (viii) that $\{P(s)-P(\bar{s})\}$ $\cap P(t)=\phi$, and as a consequence, the predecessors of the even vertices of $P(t)$ are not altered. Hence

${ }^{5}$ This argument is based on the following simple lemma: Let $\mathrm{C}$ be a closed path in a graph $\mathrm{G}$. If $\mathrm{C}$ passes through the vertex $\mathrm{x}$ exactly once, then $\mathrm{C}$ contains a circuit through $\mathrm{x}$.

$$
\tilde{P}(t)=P(t) \text {. }
$$

The third piece of $\tilde{P}(v)$ is therefore simple. The same holds for the two other pieces by virtue of (vi), and since the reverse of the path $\tilde{P}(w)-\tilde{P}(t)$ is part of the simple path $P(s)-P(\bar{s})$ by defirition of $B$. Thus the proof of the lemma reduces to verifying

(x)

$$
\begin{aligned}
& \{\tilde{P}(w)-\tilde{P}(t)\} \cap P(t)=\phi \\
& \{P(v)-P(w)\} \cap P(t)=\phi \\
& \{P(v)-P(w)\} \cap\{\tilde{P}(w)-\tilde{P}(t)\}=\phi .
\end{aligned}
$$

Now (x) follows from (vii) and (viii) since $\tilde{P}(w)-\tilde{P}(t)$ $\subseteq P(s)-P(\bar{s})$. To prove (xi), we note that $w \in \bar{P}(s)$ $-\bar{P}(\bar{s}) \subseteq H_{x}$. Hence $(\bar{s}, x)$ bisects $P(w)$. Since $P(v) \supseteq \bar{P}(w),(s, x)$ bisects $P(v)$ also, and we have

$$
P(v)-P(w) \subseteq H_{x} .
$$

This, together with (vii) proves (xi).

Finally assume $\quad z \epsilon\{P(v)-P(w)\} \cap\{\tilde{P}(w)-\tilde{P}(t)\}$. Without restriction of generality we may assume that $z$ is even in $P(v)$; otherwise we replace $z$ by $m(z)$. $z$ occurs in $P(s)-P(\bar{s})$ since $\tilde{P}(w)-\tilde{P}(t) \subseteq P(s)-P(\bar{s})$. If $z$ is even in $P(s)$, we have $w \leqslant s$ as a consequence of $w \leqslant z$ and $z \leqslant s$. But this contradicts the fact that $w$ is odd in $P(s)$. If, on the other hand, $z$ is odd in $P(s)-P(\bar{s})$, then $w$ would not be the first even vertex of this kind in $\tilde{P}(v)$. This proves (xii) and completes the proof of lemma 3 .

\section{Algorithms $\mathfrak{T}$ and $\mathfrak{M}$}

We proceed to describe two algorithms which are based on successively enlarging labeled subgraphs by forward and blossom steps. The algorithm $\mathfrak{I}$ determines all vertices of a given matched graph $(G, M)$ that are legally accessible from a given exposed vertex $r$. It also arranges the connecting alternating paths in a treelike manner, thereby proving the main theorem. The algorithm $\mathfrak{M}$ is essentially based on the algorithm $\mathfrak{I}$, however its purpose is the construction of a maximum matching.

The algorithm $\mathfrak{I}$ goes as follows: Start out with the trivial labeled subgraph $L=(A, \Omega, I, p)$ whose graph $A$ consists of the root $r$ only. $r$ is labeled "outer", that is, $\Omega=\{r\}$ and $I:=\phi$. The predecessor function need not be defined since its region of definition $\Omega-\{r\}$ is vacuous. Then enlarge $L$ successively by forward and blossom steps until a subgraph is reached that permits no further enlargement. We call such a labeled subgraph terminal. A terminal subgraph must be reached eventually since each step increases the number of edges in $A$, and this number is bounded by the number of edges in $G$. Terminal labeled subgraphs correspond to Hungarian trees (Edmonds [1]), if $M \cap A$ is part of a maximum matching.

LEMMA 4. If $\mathrm{L}=(\mathrm{A}, \Omega, \mathrm{I}, \mathrm{p})$ is terminal, then the vertices in $\Omega$ are precisely those vertices that are legally accessible from $\mathrm{r}$. 
Proof: Let $v \notin \Omega$ be a vertex which is joined to $r$ by a simple even alternating path $V$. It is no restriction of generality to assume that all other even vertices of $V$ belong to $\Omega$. Denote by $w$ the even vertex in $V \cap \Omega$ closest to $v$. Thus $(v, m(v), w)$ is a bi-edge. Now we treat two cases.

CASE 1: $v \notin I$. Then $v \notin \Omega \cup I$ and so $m(v) \notin \Omega \cup I$ (property (i)). Hence the bi-edge $(v, m(v), w)$ can be added to $L$ (forward step), contradicting the fact that $L$ is terminal.

CASE 2: $v \epsilon I$. Hence $m(v) \epsilon \Omega$ according to (ii), and deleting the edge $(v, p m(v))$ decomposes $A$ into two components $H_{v}$ and $H_{r}$ according to (iv). The edge of $V$ meeting $v$ is $(v, m(v))$, which leads the path $V$ into $H_{v}$. Since $V$ has no double points, it cannot contain the edge $(v, p m(v))$. Therefore, since $(v, p m(v))$ alone joins $H_{v}$ to the rest of $A$ and since $r$ is not in $H_{v}, V$ must leave $A$ eventually. Let $(z, u)$ with $z$ in $A$ be the first edge of $V$ after $v$ not in $A$. By virtue of property (i), the edge $(z, u)$ is not matched. Hence $z$ is odd, and $u$ is even in $V$. Since $u \neq v$, we have $u \epsilon \Omega$ by hypothesis. This implies $z \epsilon I$, because otherwise $z$ and $u$ would define a blossom containing $v$ showing $L$ to be not terminal.

The part of the path $V$ between $w$ and $z$, but in reverse order, forms together with $P(m(v))$ the path.

$$
Z=(z, m(z), \ldots, w, P(m(v))) \subseteq A,
$$

that joins $z$ to the root $r$. $Z$ does not contain the edge $(z, p m(z))$. Otherwise, $z$ would occur twice in $Z$, and this is impossible since $(z, m(z), \ldots, w)$ is simple as part of $V$, and since $z \epsilon H_{v}$ and $P(m(v)) \cap H_{v}=\{m(v), v\}$. The existence of $Z$ contradicts the fact that, according to (iv), the edge $(z, p m(z))$ separates $z$ from $r$ in $A$. Thus there are no legally accessible vertices outside $\Omega$. That all vertices of $\Omega$ are indeed legally accessible follows by $(\mathrm{v})$. This completes the proof of the lemma.

Algorithm $\mathfrak{I}$ proves our theorem for exposed roots $r$. However, as we already pointed out in section 2, this is sufficient to prove the theorem for general roots.

Algorithm $\mathfrak{M}$ starts out with any matched graph $(G, M)$. If there are no exposed vertices, then the matching is maximum. Otherwise choose an exposed vertex $e$ as a root, and employ algorithm $\mathfrak{I}$ to find outer vertices connected to $e$. If some outer vertex $v$ is adjacent to an exposed vertex $f$ different from $e$, then the edge $(f, v)$ together with the backpath $P(v)$ forms an augmenting path. Replacing matched edges by unmatched ones, and vice versa, along this path yields a new matching $M$ of higher cardinality. Algorithm $\mathfrak{I}$ then is repeated with one of the remaining exposed vertices as roots.

If algorithm $\mathfrak{I}$ does not produce an augmenting path, then we consider the terminal labeled subgraph $A$ and the graph $\tilde{G}:=G-A$ that results by deleting from $G$ all vertices of $A$ and all edges adjoining $A$. $\tilde{G}$ is again a matched graph, and algorithm $\mathfrak{M}$ is applied to it. This procedure is justified by the following lemma due to Edmonds [1]:

LEMMA 5. If $\mathrm{A}$ is a terminal labeled subgraph none of whose outer vertices are joined to an exposed vertex by an edge not in $\mathrm{A}$, then the matching $\mathrm{M}$ of $\mathrm{G}$ is maximum if and only if its restriction to $\mathrm{G}-\mathrm{A}$ is maximum.

Proof: The nontrivial direction is to show that if $M$ is not maximum, then neither is its restriction to $G-A$. If $e$ is the $\operatorname{root}$ of $A$, then $e$ is not the endvertex of an augmenting path. Indeed, if there exists an augmenting path $P$ joining $e$ to an exposed vertex $f$, then the vertex $v$ which is next to $f$ in $P$ is legally accessible from $e$. Hence $v \epsilon \Omega$ according to lemma 4 . On the other hand, $f \notin A$, and therefore $(v, f)$ is not an edge of $A$, contradicting the hypothesis of the lemma.

Now let the augmenting path $V$ join the two exposed vertices $f$ and $g$, both in $G-A$. We have to show

(xiii)

$$
V \subseteq G-A .
$$

Assume $V \cap A \neq \phi$. Since $x \epsilon V \cap A$ implies $m(x) \epsilon V \cap A$, we conclude $V \cap \Omega \neq \phi$. Let $v \epsilon V \cap \Omega$, then there exists a back-path $P(v)$ connecting $v$ and $e$. Let $w$ be the vertex in $P(v) \cap V$ which is closest to $e$ in $P(v)$. w is odd in $P(v)$; otherwise, $m(w) \in P(v) \cap V$ would be closer to $e$ in $P(v)$. $\quad w$ divides the path $V$ into two pieces; the piece which contains $m(w)$, forms together with $P(m(w))$ an augmenting path ending at $e$. But such an augmenting path has been shown not to exist. This proves (xiii), and thereby the lemma.

\section{An Example by E. Johnson}

Berge [4] considers a generalization of the maximum matching problem. Given for each vertex $v$ of a graph $G$ a nonnegative integer $d(v)$, one calls a degreeconstrained subgraph a subgraph $M \subseteq G$ whose degrees at each vertex $v$ are bounded above by $d(v)$. The problem is to find a degreeconstrained subgraph with a maximum number of edges. The maximum matching problem results if $d(v)=1$ for all vertices $v$ of $G$. Maximum degreeconstrained subgraphs are again characterized by the absence of "augmenting paths". Augmenting paths are again alternating paths without multiple edges; multiple points however are permitted (Berge [4], compare also Goldman [6]).

Recently, Edmonds [3] extended his algorithm to an efficient solution algorithm for the above and even more general problems. This leads to the question of extending the predecessor technique described in this paper.

Alternating paths and bi-edge graphs can be defined for every pair $(G, S)$, where $S$ is any set of edges of $G$, not necessarily a matching. The theory of degreeconstrained subgraphs suggests defining legal alternating paths as alternating paths without multiple edges, but possibly multiple vertices. The predecessor technique now requires that our theorem holds for general $S$ with the above concept of legality.

This conjecture is refuted by the following example due to E. Johnson. The graph $G$ of this example is presented in figure 7; heavy lines distinguish the edges in $S$.

Choosing vertex $l$ as the root, all vertices are legally accessible by alternating paths of even length. From 
vertex 2 , for instance, the root can be reached by the paths $(2,3,5,4,3,6,7,8,9,10,8,2,1)$ or $(2,8,10,9,8$, $7,6,3,4,5,3,2,1)$. Up to interchanges of 4 with 5 and 9 with 10 , these are the only simple alternating paths joining 2 to the root. If the first path is to appear in a tree $T \subseteq B(G, S)$, then the predecessor of 8 must be 6 . But up to an interchange of 9 with 10 , the only back-path of 7 is $(7,8,10,9,8,2,1)$ requiring $l$ to be the predecessor of 8 . A similar contradiction appears if the second back-path of 2 is chosen to be in the tree. Thus the legal back-paths in figure 7 cannot be arranged in a treelike manner. Without the legality requirement, however, no difficulty would have arisen.

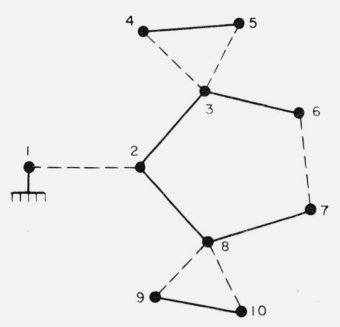

FigURE 7
This example proves that the maximum degreeconstrained subgraph problem cannot be solved by a direct extension of the predecessor algorithm. Another generalization of the maximum matching problem, also solved by Edmonds [2], consists in maximizing a weighted sum of the edges. The shrinking technique of Edmonds appears to be more natural for this problem also.

\section{References}

[1] Edmonds, J., Paths, trees, and flowers (to appear, Canadian J. Math. (May 1965).

[2] Edmonds, J., Maximum matching and a polyhedron with 0, $1-$ vertices, J. Res. NBS 69B (Math. and Math. Phys.) Nos. 1 and 2, 125-130 (Jan.-June 1965).

[3] Edmonds, J., Optimum degreeconstrained subgraphs (in preparation).

[4] Berge, C., The Theory of Graphs and Its Applications (English translation from French), John Wiley \& Sons, Inc., New York, N.Y.).

[5] Edmonds, J., Covers and Packings in a Family of Sets, Bull. Amer. Math. Soc. 68, No. 5, (Sept. 1962).

[6] Goldman, A. J., Optimal matchings and degreeconstrained subgraphs, J. Res. NBS 68B (Math. and Math. Phys.), No. 1, 27-29 (1964). 